\title{
Eficacia de estrategias de disminución del calentamiento urbano. Estudio para una ciudad de clima árido
}

\section{Effectiveness of strategies to reduce urban warming. Study in a city with arid climate}

María Belén Sosa ${ }^{(*)}$, Erica Correa ${ }^{(*)}$, María Alicia Cantón ${ }^{(*)}$

\section{RESUMEN}

Las ciudades modifican los parámetros microclimáticos aumentando el calentamiento urbano; en virtud de ello, existen diversas estrategias que colaboran a disminuir este impacto en contextos áridos. El presente trabajo evalúa el nivel de eficacia de dos estrategias factibles de ser incorporadas. Para ello, se diseñaron 96 escenarios que combinan 2 alturas de edificación (3 y $6 \mathrm{~m}), 3$ anchos de calle $(16,20$ y $30 \mathrm{Om})$ y 4 orientaciones (E-O, N-S, NO-SE y NE-SO). Mediante el software ENVI-met se simuló el comportamiento térmico de los escenarios propuestos, el modelo fue validado mediante el monitoreo microclimático in situ. Las etapas de simulación fueron: 1.morfología, 2.forestación, 3.albedo y 4.estrategias combinadas. Los hallazgos más importantes indican que la incorporación de las estrategias combinadas resulta ser la opción más eficaz ya que se potencian los beneficios individuales; alcanzando disminuciones de hasta un $12 \%$ en la temperatura máxima, $10 \%$ en la mínima y $11 \%$ en la promedio.

Palabras clave: Calentamiento urbano, diseño urbano, estrategias de mitigación, eficacia.

\section{ABSTRACT}

Cities modify microclimatic parameters that increase urban warming. In regard to this, there are some strategies that contribute to the reduction of this impact in arid contexts. The present work evaluates the level of effectiveness of strategies that can be feasible incorporated. For this, 96 scenarios that combine 2 building heights (3 and 6m), 3 street widths (16, 20 and 3om) and 4 orientations (E-O, N-S, NO-SE and NE-SO) were designed. By using ENVI-met software, the thermal behavior of the proposed scenarios was simulated; the model was validated by in situ microclimatic monitoring. The simulation stages were: 1.morphology, 2.forestation, 3.albedo and 4.combined strategies. The most important findings indicate that the incorporation of combined strategies is the most effective option that maximizes the individual benefits; reaching decreases of up to $12 \%$ in the maximum temperature, $10 \%$ in the minimum and $11 \%$ in the average.

Keywords: Urban warming, urban design, mitigation strategies, effectiveness.

(*) Instituto de Ambiente, Hábitat y Energía (INAHE), Consejo Nacional de Investigaciones Científicas y Técnicas (CONICET), CCT-Mendoza, Argentina.

Persona de contacto/Corresponding author: msosa@mendoza-conicet.gob.ar (M. B. Sosa).

ORCID: http://orcid.org/oooo-0002-0141-442X (M. B. Sosa); http://orcid.org/oooo-0003-1690-076X (E. Correa);

http://orcid.org/oooo-0002-8714-9697 (M. A. Cantón).

Cómo citar este artículo/Citation: Sosa, María Belén; Correa, Erica; Cantón, María Alicia (2020). Eficacia de estrategias de disminución del calentamiento urbano. Estudio para una ciudad de clima árido. Informes de la Construcción, 72(559): e352. https://doi.org/10.3989/ic.66662.

Copyright: (C) 2020 CSIC. Este es un artículo de acceso abierto distribuido bajo los términos de la licencia de uso y distribución Creative Commons Reconocimiento 4.0 Internacional (CC BY 4.0). 


\section{INTRODUCCIÓN}

Las ciudades experimentan desde el siglo XIX diversos procesos de re-estructuración, regidos por los cambios dinámicos de orden económico y social a los cuales las áreas urbanas se fueron adaptando mediante una acelerada expansión territorial (1). A partir de la década de los '70-80, se consolidan los modelos de desarrollo urbano extensivos, en desmedro de continuar con la densificación de las zonas urbanas consolidadas y del centro histórico. El modelo de expansión territorial de las áreas urbanas sobre las zonas periféricas, es conocido como "disperso" o con su denominación en inglés como "urban sprawl". Este esquema de desarrollo consume muchos recursos y genera importantes consecuencias negativas sobre el territorio. La dispersión aumenta los costos asociados a la ejecución y el mantenimiento de todas las redes de abastecimiento e infraestructuras urbanas, disminuye el aprovechamiento del suelo disponible, aumenta las externalidades asociadas a los medios de transporte y limita las capacidades de regulación del planeamiento urbano (2). Esta forma de expansión de las áreas urbanas, ha favorecido la pérdida de habitantes en las ciudades, siendo la baja densidad de población un indicador de dispersión. El último informe mundial de las ciudades de la ONU, indica que la expansión demográfica y espacial están directamente relacionadas; estimando que una disminución del $1 \%$ en la densidad, entre los años 2000 y 2050, cuadruplicaría la superficie que ocupan las ciudades a nivel mundial (3). Este factor contribuye a que una misma población ocupe una superficie mayor de territorio, generando que el uso del suelo urbanizado como recurso sea menos eficiente. Siendo el territorio sobre el cual se desarrollan las áreas urbanas un recurso natural, escaso y difícil de renovar para otros usos (4).

La forma de crecimiento y disposición de esta expansión de superficie urbanizada impacta, entre otros factores, sobre las condiciones climáticas del sitio de inserción. Estas alteraciones sobre los patrones climáticos generan condiciones ambientales concretas que se pueden definir como "microclima urbano" (5). Entre los mayores impactos sobre los patrones microclimáticos se destaca el efecto de calentamiento urbano (6). El calentamiento de las áreas urbanas está determinado por flujos superficiales que modifican los valores de temperatura y humedad (7). En este sentido, la forma, tamaño y disposición de la ciudad, la contaminación atmosférica, el calor generado por diversas fuentes antropogénicas, las propiedades termofísicas de las superficies urbanas, la sustitución de vegetación con superficies impermeables y el aumento de la rugosidad, son los factores que comúnmente alteran las variables microclimáticas $(8,9,10)$.

En la actualidad, el reto de alcanzar desarrollos urbanos sostenibles, demanda generar nuevos planteamientos para planificar las áreas urbanas (2). Entendiendo a la sostenibilidad como un equilibrio dinámico, en el cual las metas se van articulando a mediano y largo plazo, en base a los condicionantes propios de cada ciudad (11). A escala local, la planificación urbana procura mejorar la calidad de vida de las personas, maximizando de manera eficiente el uso de los recursos naturales disponibles; controlando y minimizando el impacto que ejerce la ciudad sobre el medio ambiente y el clima. En este sentido, generar y transformar conocimiento científico en herramientas de planificación que sean aplicables al diseño y construcción de ciudades más sostenibles a nivel local se considera una estrategia de alto impacto $(12,13,14,15,16$, 17, 18).
En torno a este eje de pensamiento, el presente trabajo busca evaluar el nivel de eficacia de diversas estrategias de reducción de las temperaturas urbanas en relación a disminuir el impacto térmico - calentamiento urbano- en el área Metropolitana de Mendoza, Argentina.

\subsection{Antecedentes}

A mediados de la década del 80', varias investigaciones llevadas a cabo en diversas regiones han permitido desarrollar una amplia variedad de estrategias para contrarrestar el impacto microclimático que genera el aumento de la temperatura en las áreas urbanas $(19,20,21,22,23)$.

Rizwan et al. (24), categorizan a las estrategias en relación a: (i) controlar los mecanismos de intercambio de energía y (ii) evaluar la etapa de planificación urbana en la que se encuentre el área de intervención. Esta diferenciación y categorización es de importancia para adecuar la inclusión de determinadas estrategias de mejora. En cuanto a "controlar los mecanismos de intercambio de energía”, los autores mencionan las siguientes estrategias: diseño y materialidad de los elementos de la envolvente urbana (techos verdes, envolventes con altos niveles de albedo, enfriamiento por pulverización de techo, etc.); reducción del calor antropogénico liberado (menor uso de aires acondicionados, disminución del tráfico urbano, etc.); otros factores tecnológicos (humidificación, paneles fotovoltaicos, pinturas termo-crómicas, etc.). Y, en correspondencia con "evaluar la etapa de planificación urbana en la que se encuentre el área de intervención”, se diferencian en: propuestas que sólo pueden ejecutarse durante la fase de diseño (factor de visión de cielo - SVF, selección de materiales de construcción, etc.) y medidas de rehabilitación que se pueden aplicar en entornos urbanos existentes (enverdecer las áreas parquizadas, enfriamiento por pulverización en techo, pinturas o revestimientos reflectivos en fachadas). Por otra parte, Kleerekoper et al. (25), recopilan y estudian los impactos de algunas estrategias de reducción de las temperaturas diferenciadas en cuatro categorías, siendo estas: forma urbana, materiales de las envolventes urbanas, vegetación y agua.

En cuanto a la forma urbana, Jusuf et al. (26), NRDC et al. (27), indican que un barrio bien planificado que considere el diseño del conjunto, las alturas de la edificación, las dimensiones y orientaciones de las calles y la forma de las manzanas; puede reducir los impactos sobre el microclima y mejorar la habitabilidad térmica. Los resultados entre las numerosas investigaciones que discuten cómo ciertas variables de la forma urbana colaboran a reducir las temperaturas exteriores indican que la magnitud del impacto de las diversas estrategias utilizadas no es igual; siendo su eficacia dependiente de las particularidades climáticas y de la forma de cada ciudad de estudio (28, 29, 30). En una ciudad de clima árido Bourbia \& Awbi $(31,32)$ estudiaron el efecto que ejercen los indicadores $\mathrm{H} / \mathrm{W}$, SVF y la orientación de la calle, en las temperaturas de un conjunto de canales urbanos. Concluyeron que controlando la geometría $(\mathrm{H} / \mathrm{W}$ y SVF) y la orientación es posible prevenir las altas temperaturas; recomiendan que en canales anchos $(\mathrm{H} / \mathrm{W}<0.5)$ se deben introducir árboles para mejorar la condición térmica.

Por otra parte, aumentar los valores de albedos de los materiales de las envolventes urbanas e incorporar vegetación en zonas urbanas permite disminuir la temperatura del aire. Middel et al. $(33,34)$, han evaluado los beneficios combinados mediante la incorporación de techos "fríos" y árboles a escala 
de barrio. Sus resultados demuestran que un aumento del 25\% de la copa de los árboles en barrios residenciales disminuye en promedio $2^{\circ} \mathrm{C}$ la temperatura durante el día, mientras que los techos fríos reducen sólo $0.3^{\circ} \mathrm{C}$. A su vez, los forestales en los canales urbanos muestran un potencial de enfriamiento promedio de 1 a $4.7^{\circ} \mathrm{C}$ que se extiende de 100 a $1000 \mathrm{~m}$ Schmidt, (35); y un equivalente a $20-30 \mathrm{~kW}$ de potencia (comparable a la potencia de 10 unidades de aire acondicionado) en un día soleado por los procesos de evapotranspiración (36). Por último, la incorporación de agua en el espacio urbano funciona como amortiguador de calor; siendo en general más eficaces cuando tienen una gran superficie, o cuando el agua está en movimiento, como una fuente. Nishimura et al. (37) y Barakat, et al. (38), mencionan que este recurso tiene un efecto de enfriamiento promedio entre $1 \mathrm{a} 3^{\circ} \mathrm{C}$, con un impacto en una extensión de superficie aproximada entre 30 a $35 \mathrm{~m}$.

La bibliografía citada muestra un compendio de las estrategias que han sido empleadas internacionalmente con el fin de reducir el calentamiento urbano y sus impactos asociados. Es importante identificar cuáles de estas estrategias son las más aptas para una determinada condición climática; como así también la etapa de planificación en que se encuentra el sector urbano a intervenir, ya que varias estrategias y tecnologías solo pueden ser empleadas para nuevos planes de diseño urbano asociadas a la disponibilidad de recursos con la que se dispone -naturales, económicos, industriales, sociales, etc. - para tal fin. Todhunter (39), toma la postura de que a microescala, los aportes de la forma urbana en la mejora térmica del espacio exterior son más importantes que el efecto del albedo de los materiales. Considerando que el potencial de sombreado de la masa urbana es más relevante para la mejora de las condiciones térmicas exteriores que las características superficiales del canal vial urbano. Asimismo, la vegetación tiene grandes potencialidades de ser incorporada en los nuevos desarrollos, como en los esquemas urbanos existentes mediante cuatro tipos diferentes de aplicación: bosques urbanos (parques), forestales de alineación, verde en patios privados $\mathrm{y}$, techos y fachadas verdes (25). Por otra parte, las técnicas de enfriamiento evaporativo demuestran su eficacia, pero no constituyen una herramienta sustentable para ciudades emplazadas en zonas áridas, por la escasez del recurso hídrico.

El avance del campo científico ha permitido incorporar el uso de métodos numéricos y físicos para modelizar y pronosticar como ciertas variables y características urbanas modifican y alteran a escala micro el clima urbano; siendo este avance considerado como una gran ventaja sobre las mediciones in situ (28). Según el estudio de Mirzaei \& Haghighat, (40), las técnicas para estudiar el microclima se pueden dividir en dos grupos: "aproximaciones observacionales" que pueden ser mediciones de campo, teledetección térmica o modelización a pequeña escala (modelos construidos para pruebas en túnel de viento); y "enfoques de simulación", que pueden ser modelos de balance energético o estudios numéricos utilizando la Dinámica Computacional de Fluidos (CFD).

La principal ventaja de los estudios de simulación en comparación con los observacionales es la posibilidad de realizar análisis comparativos basados en diferentes escenarios. Por otra parte, el principal inconveniente de los "enfoques de simulación" es la necesidad de aplicar varias simplificaciones, ya que la física subyacente al microclima urbano es muy compleja. Por lo tanto, la validación cuidadosa de los resultados obtenidos mediante simulaciones es muy importante. A me- dida que el uso de las herramientas de CFD aplicadas a la física urbana sigue ganando popularidad, se han publicado varias directrices para mejorar la calidad de las simulaciones y limitar los errores de modelado numérico y físico (41, 42, 43). Las simulaciones CFD pueden emplearse para estudiar el clima a diferentes escalas espaciales, siendo estas escalas: mesoescala meteorológica $(<200 \mathrm{~km})$, microescala meteorológica $(<2 \mathrm{~km})$, edificios $(<100 \mathrm{~m})$ e interior de edificios (<10m) (44). Los softwares CFD de microclima urbano varían sustancialmente en función de su base física, su resolución espacio-temporal y las variables de salida o "outputs" (45). Siendo los más utilizados: ENVI-met, SOLENE-Microclimate, Townscope, entre otros. De manera particular ENVI-met ha sido validado científicamente en numerosas publicaciones (46, 47, 48 y 49 entre otros), por reflejar la capacidad del modelo para calcular con precisión las variables microclimáticas a través de numerosas métricas reportadas y diversos índices aplicados para la evaluación de este modelo (50).

\subsection{Modelo urbano del área de estudio}

Las ciudades desarrolladas en ambientes áridos, donde la condición hídrica es la principal limitante suelen presentar dos tipos de modelos urbanos cuyas características son diferenciales: modelo compacto y modelo abierto. Las ciudades de "modelo compacto" como el caso de Argel (Argelia) o Najran (Arabia Saudita), presentan un desarrollo urbano continuo, edificios altos y calles estrechas, donde el uso de forestación es inexistente o escaso. Desde el punto de vista microclimático, el modelo en sí mismo construye sombras que disminuyen la exposición solar y, en consecuencia, la acumulación de calor sobre las superficies expuestas. Por otra parte, el "modelo abierto" - como es el área Metropolitana de Mendoza (AMM) - tiene calles anchas y construcciones relativamente bajas, enmarcadas por un entramado de árboles que le han otorgado la denominación internacional de "ciudad oasis" (51). El objetivo de ambos modelos urbanos se basa en acondicionar ambientalmente un sector de desierto para el desarrollo de las actividades humanas, la Fig. 1 muestra imágenes de ambos casos.
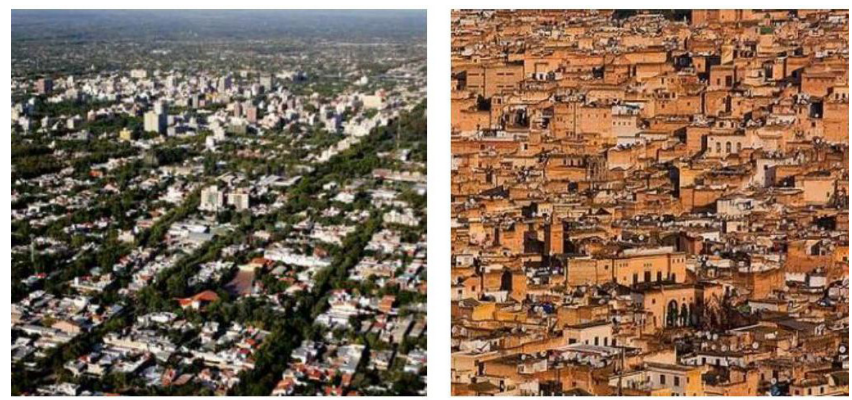

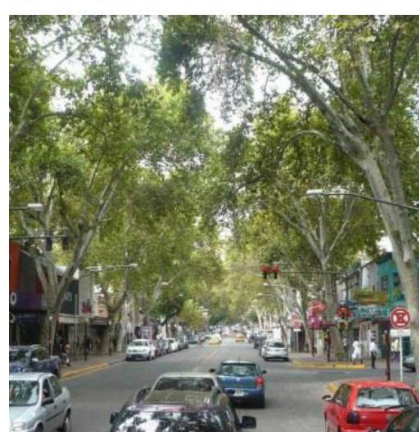

Modelo abierto - Mendoza

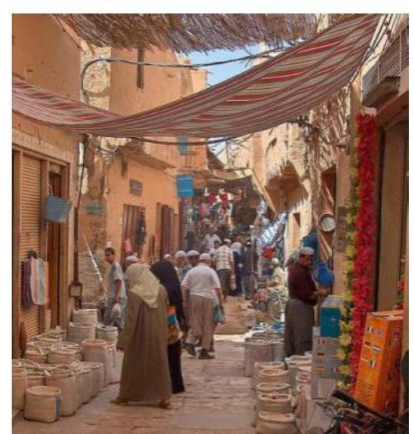

Modelo compacto - Argel
Figura 1. Modelos de ciudad abierto y compacto. 
Respecto a las densidades edilicias, dentro del sector central del AMM se encuentran los desarrollos de mayor ocupación de suelo, descendiendo gradualmente hacia la periferia. En las décadas del 80’ y muy especialmente la del 90' el área metropolitana creció más del doble del total provincial, siendo períodos de fuerte concentración de población en el área urbana. Esta expansión urbana se ha reflejado en el crecimiento de la baja densidad edilicia, que según datos del Instituto Provincial de la Vivienda está conformada en un 99\% por viviendas unifamiliares de 1 o 2 pisos (52).

\section{METODOLOGíA}

\subsection{Selección caso de estudio}

El AMM está conformado por 6 departamentos: Capital, Las Heras, Godoy Cruz, Guaymallén, Maipú y Luján de Cuyo. Con el objetivo de delimitar el área de estudio dentro del AMM, se seleccionó uno de los departamentos de mayor ocupación demográfica Las Heras (19\%), (53). Dentro del departamento se seleccionó un barrio mediante un relevamiento que caracterizó y cuantificó los tipos de tramas urbanas existentes. Esta tipificación morfológica se realizó utilizando un plano catastral y el software AutoCADß. Las manzanas se diferenciaron de acuerdo a 3 formas: (i) damero, cuatro lados de iguales dimensiones, color lila; (ii) rectangular de mayor exposición en eje Norte-Sur, color celeste o Este-Oeste, color naranja; y (iii) irregular, tienen cuatro o más lados de diferentes longitudes, color magenta. Los resultados del relevamiento gráfico indican que en Las Heras las formas más representativas son la irregular (33\%) seguida por la rectangular con orientación Norte-Sur (30\%). A partir de este relevamiento se seleccionó un barrio donde la característica morfológica del conjunto son las manzanas rectangulares orientadas en sentido Norte-Sur. Dentro de esta trama se escogió un canal vial para monitorear su comportamiento térmico. La morfología del canal vial se puede caracterizar con descriptores e indicadores urbanos, siendo: largo de calle 192m, ancho de calle $16 \mathrm{~m}$, orientación de calle Norte-Sur, altura de viviendas $3 \mathrm{~m}, \mathrm{H} / \mathrm{W}$ o.18, viviendas con pintura y techos con revestimiento cerámico, especie forestal Morus alba 10m. En la Fig. 2 se muestra el mapa del relevamiento gráfico con su respectiva codificación por color, la planimetría del barrio con el punto de monitoreo térmico, una fotografía de la sección del canal vial seleccionado y la fachada del prototipo de vivienda del barrio.

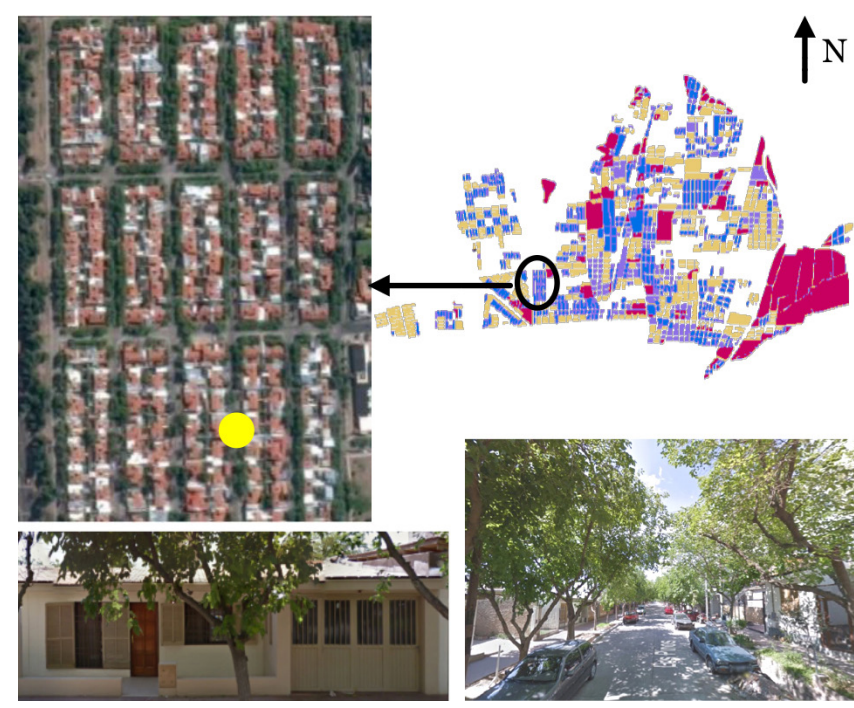

Figura 2. Caso de estudio.

\subsection{Monitoreo térmico y obtención del SVF}

El monitoreo y obtención de los datos microclimáticos se realizó desde el de 8 enero hasta el 10 febrero de 2014 (32 días corridos). En el canal vial seleccionado se instaló en la vereda con frente Este, a una altura de $2 \mathrm{~m}$, un sensor fijo tipo Ho8003-02 sobre un poste de madera con un precinto plástico. El sensor se colocó dentro de una caja blanca de PVC perforada, con el objeto de evitar la irradiación y asegurar una adecuada circulación de aire (54). Las mediciones de temperatura y humedad relativa fueron programadas cada 15 minutos. El SVF fue calculado a partir de imágenes hemisféricas capturadas en el centro del canal vial con una cámara digital equipada con un lente ojo de pez; procesadas con el software Píxel de Cielo (55). Siendo este indicador urbano de gran importancia para ciudades como el área de estudio que poseen forestación de alineación en sus calles, como se visualiza en la sección del canal vial, Fig. 2.

\subsection{Modelo físico de simulación. Descripción y validación}

El software ENVI-met v3.1 fue seleccionado para realizar las simulaciones microclimáticas. ENVI-met trabaja con una serie de datos de entrada o "inputs", siendo estos datos diferenciados en tres componentes:

- Area Input File, se crea a través de una interfaz gráfica, se replica la geometría y forestación de las condiciones reales del caso de estudio donde se tomaron las mediciones microclimáticas. Este modelo fue realizado en una versión $100 \times 100$ x 30, resolución de 3 × 3 × 3m y número de grillas x: 86; y: 86; z: 30. Se colocaron un total de 3 receptores, uno de ellos se ubicó en el centro del área de estudio, y los otros dos se ubicaron en las esquinas del canal vial.

- Database, se refiere a los datos y características de elementos como forestación y estratos del suelo. El árbol utilizado fue el "Tb" de la librería PLANTS.DAT de ENVI-met, la altura se determinó en 10m por corresponder con el desarrollo promedio de un forestal de $2^{\circ}$ magnitud como el caso de Morus alba. Las características de esta tipología forestal son: 400 de resistencia mínima estomática, 0.20 de albedo de onda corta de la hoja de la planta, $2 m$ de profundidad total de la zona radicular, LAD de 0.80 a 2.00 de densidad del área foliar en $\mathrm{m}^{2} / \mathrm{m}^{3}$ y RAD de 0.10 de densidad del área de la raíz en $\mathrm{m}^{2} / \mathrm{m}^{3}$

- Configuration File, se ingresan parámetros de cálculo y condiciones de contorno que caracterizan la simulación. En este archivo se indican todos los datos iniciales de configuración de la simulación a realizar, como así también los datos climáticos iniciales, la tabla 1 muestra los datos y valores utilizados.

Con el fin de validar los resultados se realizó un ajuste entre el comportamiento microclimático del caso real (monitoreado in situ) con el modelo físico. Para ello se escogió uno de los días monitoreados durante el año 2014 que representa las condiciones del total del periodo (18/01/2014); día claro, despejado y de elevada heliofanía. El periodo de estabilización encontrado en esta investigación de 72 horas, es congruente con los resultados obtenidos por Wang \& Akbari (56), quienes también toman como salida válida sólo aquellos valores de las últimas 24 horas para reproducir correctamente la fenomenología que caracteriza el día elegido. 
Tabla 1. Archivo de configuración, configuration file.

\begin{tabular}{|c|c|}
\hline Meteorológicos & Valores \\
\hline Velocidad viento a $10 \mathrm{~m}$ sobre suelo $(\mathrm{m} / \mathrm{s})$ & 2.5 \\
\hline Dirección viento $\left(0^{\circ}: \mathrm{N}, 90^{\circ}: \mathrm{E}, 180^{\circ}: \mathrm{S}, 270^{\circ}: \mathrm{W}\right)$ & $140^{\circ}$ \\
\hline Rugosidad $\mathrm{z}_{\mathrm{o}}$ & 0.1 \\
\hline Temperatura atmosférica inicial (K) & 300 \\
\hline Humedad específica a 2500m.s.n.m (g/kg-1) & 2.8 \\
\hline Humedad relativa a $2 \mathrm{~m}(\%)$ & 37 \\
\hline \multicolumn{2}{|l|}{ Edificación } \\
\hline Temperatura interior $(\mathrm{K})$ & 297 \\
\hline Conductividad pared $\left(\mathrm{W} / \mathrm{m}^{2} \mathrm{~K}\right)$ & 2 \\
\hline Conductividad techo (W/m²K) & 0.78 \\
\hline Albedo pared & 0.3 \\
\hline Albedo techo & 0.2 \\
\hline \multicolumn{2}{|l|}{ Suelo } \\
\hline Temperatura superficial, capa inicial $(0-20 \mathrm{~cm})$ & $292 K^{*}$ \\
\hline Temperatura superficial, capa media $(20-50 \mathrm{~cm})$ & $292 K^{*}$ \\
\hline Temperatura superficial, capa profunda $(<50 \mathrm{~cm})$ & $290 K^{*}$ \\
\hline Humedad relativa, capa inicial (o-20cm) & $20 \% *$ \\
\hline Humedad relativa, capa media $(20-50 \mathrm{~cm})$ & $35 \% *$ \\
\hline Humedad relativa, capa profunda $(<50 \mathrm{~cm})$ & $60 \% *$ \\
\hline
\end{tabular}

*valores por defecto de ENVI-met

La tabla 2 muestra las curvas de temperatura del aire simulada y medida, los valores de SVF, como así también los indicadores estadísticos de ajuste. Donde $\mathrm{R}^{2}$ es el coeficiente de correlación ajustado, RSME (Root Mean Square Error) el error cuadrático medio, MBE (Mean Bias Error) el error de polarización media y MAE (Mean Average Error) el error absoluto medio. Cada indicador expresa el grado de exactitud o error del modelo bajo diversas perspectivas, MAE y RMSE indican la magnitud del error promedio, pero no proporcionan información sobre el tamaño relativo de la diferencia media entre los valores observados y los valores predichos. MBE describe la dirección del sesgo de error, su valor está relacionado con la magnitud de los valores utilizados; un MBE negativo se produce cuando las predicciones son más pequeñas en valor que las observaciones.

Tabla 2. Ajuste modelo simulado con medición in situ.

\begin{tabular}{|l|c|c|c|c|c|c|c|}
\hline 40 \\
38 \\
36 \\
34 \\
32 \\
30
\end{tabular}

\subsection{Descripción de los escenarios urbanos y etapas de simulación}

Los escenarios o modelos teóricos evaluados se desprenden de las posibilidades reales de generar estos esquemas urbanos siguiendo las directrices de la Ley de Loteo de Mendoza ( $\mathrm{N}^{\circ}$ 4341/1979). Esta ley indica anchos (16, 20 y 30m) y longitudes de calle (entre 50 a 200m). En este sentido, se decidió incorporar los 3 anchos de calle con un largo de 10om y alturas de edificación de 3 y $6 \mathrm{~m}$, debido a que se trabaja sobre la escala edilicia de baja densidad. Por último, las orientaciones analizadas en los modelos fueron 4, siendo: Este-Oeste (E-O), Norte-Sur (N$\mathrm{S})$ y rotaciones a $45^{\circ}$ en ambos sentidos conformando así las orientaciones Noreste-Suroeste (NE-SO) y Noroeste-Sureste (NO-SE). La tabla 3 muestra las características morfológicas de cada escenario según ancho de calle y altura de edificación.

Tabla 3. Características escenarios a simular.

\begin{tabular}{|c|c|c|c|c|c|c|c|}
\hline \multirow{2}{*}{\multicolumn{2}{|c|}{$\begin{array}{c}\text { Planimetría } \\
\text { escenarios }\end{array}$}} & \multicolumn{2}{|c|}{ Ancho 16m } & \multicolumn{2}{|c|}{ Ancho $20 \mathrm{~m}$} & \multicolumn{2}{|c|}{ Ancho 3om } \\
\hline & & \multicolumn{2}{|c|}{ 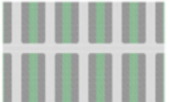 } & \multicolumn{2}{|c|}{ |110 } & \multicolumn{2}{|c|}{\begin{tabular}{l|l|}
$\|$ & $\|$
\end{tabular}} \\
\hline \multicolumn{2}{|c|}{ Área construida } & \multicolumn{2}{|c|}{$41 \%$} & \multicolumn{2}{|c|}{$38 \%$} & \multicolumn{2}{|c|}{$28 \%$} \\
\hline \multicolumn{2}{|c|}{ Área libre } & \multicolumn{2}{|c|}{$39 \%$} & \multicolumn{2}{|c|}{$35 \%$} & \multicolumn{2}{|c|}{$37 \%$} \\
\hline \multicolumn{2}{|c|}{ Área calle } & \multicolumn{2}{|c|}{$20 \%$} & \multicolumn{2}{|c|}{$27 \%$} & \multicolumn{2}{|c|}{$35 \%$} \\
\hline \multirow{3}{*}{ జ్ } & $\mathrm{H} / \mathrm{W}$ & \multicolumn{2}{|c|}{0.19} & \multicolumn{2}{|c|}{0.15} & \multicolumn{2}{|c|}{0.10} \\
\hline & \multirow{2}{*}{ SVF } & $\mathrm{SF}^{*}$ & $\mathrm{CF}^{*}$ & $\mathrm{SF}^{*}$ & $\mathrm{CF}^{*}$ & $\mathrm{SF}^{*}$ & $\mathrm{CF}^{*}$ \\
\hline & & 0.96 & 0.54 & 0.98 & 0.63 & 0.99 & 0.77 \\
\hline \multirow{3}{*}{$\begin{array}{l}\text { घี } \\
\text { 出 }\end{array}$} & $\mathrm{H} / \mathrm{W}$ & \multicolumn{2}{|c|}{0.37} & \multicolumn{2}{|c|}{0.30} & \multicolumn{2}{|c|}{0.20} \\
\hline & \multirow{2}{*}{ SVF } & $\mathrm{SF}^{*}$ & $\mathrm{CF}^{*}$ & $\mathrm{SF}^{*}$ & $\mathrm{CF}^{*}$ & $\mathrm{SF}^{*}$ & $\mathrm{CF}^{*}$ \\
\hline & & 0.77 & 0.49 & 0.82 & 0.59 & 0.90 & 0.74 \\
\hline
\end{tabular}

*SF: Sin forestación, CF: Con forestación

El total de escenarios evaluados fueron 96. Los mismos se evaluaron térmicamente en 4 etapas de simulación que varían según la incorporación estrategias:

- Etapa 1 - "escenarios base”. Evaluación puramente morfológica, combinación de 3 anchos de calle, 2 alturas de edificación y 4 orientaciones de calle, 24 escenarios.

- Etapa 2 - "escenarios forestados". Incorporación de forestales de alineación, 24 escenarios.

- Etapa 3 - "escenarios con albedos optimizados". Modificación de la tecnología de las envolventes (propiedades ópticas) de techos, muros y pisos, 24 escenarios. Los valores asignados en los techos fueron de 0.7, en los muros de $0.3 \mathrm{y}$ las superficies de piso de 0.5; siendo estos valores los recomendados para el AMM por la tesis doctoral de Alchapar, (57).

- Etapa 4 - "escenarios con estrategias combinadas". Evaluación conjunta de ambas estrategias (etapas $2+3$ ). Siendo el objetivo de esta etapa verificar si la eficacia individual de cada estrategia se potencia o disminuye al ser incorporadas de manera conjunta en un mismo entorno urbano, 24 escenarios.

\section{RESULTADOS}

\subsection{Comportamiento térmico}

La tabla 4 muestra los valores de temperatura máxima, mínima y promedio de los 96 escenarios. Cabe destacar que se analizan los valores de temperatura máxima por ser una variable que se relaciona con el grado de habitabilidad del espacio exterior, la temperatura mínima que refleja las posibilidades de enfriamiento y la temperatura promedio vinculada con el 
Tabla 4. Comportamiento térmico de los 96 escenarios.

\begin{tabular}{|c|c|c|c|c|c|c|c|c|c|c|c|c|c|c|c|c|c|c|}
\hline \multirow{2}{*}{\multicolumn{3}{|c|}{ Orientación de trama }} & \multirow{2}{*}{\multicolumn{4}{|c|}{$\begin{array}{c}\mathrm{N}-\mathrm{S} \\
\text { Etapas de simulación }\end{array}$}} & \multicolumn{4}{|c|}{ NO-SE } & \multicolumn{4}{|c|}{ E-O } & \multicolumn{4}{|c|}{ NE-SO } \\
\hline & & & & & & & \multicolumn{4}{|c|}{ Etapas de simulación } & \multicolumn{4}{|c|}{ Etapas de simulación } & \multicolumn{4}{|c|}{ Etapas de simulación } \\
\hline & & & $\mathbf{1}^{*}$ & $\mathbf{2}^{*}$ & $3^{*}$ & $4^{*}$ & $\mathbf{1}^{*}$ & $\mathbf{2}^{*}$ & $3^{*}$ & $4^{*}$ & $\mathbf{1}^{*}$ & $\mathbf{2}^{*}$ & $3^{*}$ & $4^{*}$ & $\mathbf{1}^{*}$ & $\mathbf{2}^{*}$ & $3^{*}$ & $4^{*}$ \\
\hline \multirow{9}{*}{$\begin{array}{l}\text { है } \\
\text { జت }\end{array}$} & \multirow{3}{*}{$\begin{array}{l}\text { Ancho } \\
16 \mathrm{~m}\end{array}$} & 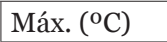 & 42.0 & 39.0 & 40.3 & 7.5 & 40.7 & 38.5 & 39.3 & 37.2 & 42.2 & 39.3 & 40.5 & 37.7 & 42.5 & 39.4 & 40.9 & 37.8 \\
\hline & & Mín. & 7.6 & $25 \cdot 3$ & $27 \cdot 3$ & 5.1 & 8.1 & 25.9 & & & 27.8 & & 27.6 & $25 \cdot 3$ & & & 27.6 & 25.8 \\
\hline & & Prom. $\left({ }^{\circ} \mathrm{C}\right)$ & 3.9 & 0 & 330 & & .5 & & & & 4 & & .5 & & & & & 0.9 \\
\hline & \multirow{3}{*}{$\begin{array}{l}\text { Ancho } \\
20 \mathrm{~m}\end{array}$} & & & & 40.0 & & & & & & & & & 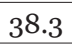 & & & & 8.5 \\
\hline & & Mín & 7.6 & 257 & 27.4 & 25 & 8.1 & 26.4 & 270 & 0 & 7.9 & 25.9 & 27.7 & $25 \cdot 7$ & 9 & & 7.7 & 26.0 \\
\hline & & & & & & & & & & & & & & & & & & \\
\hline & \multirow{3}{*}{$\begin{array}{l}\text { Ancho } \\
30 \mathrm{~m}\end{array}$} & & & 5 & 38 & & & 40.0 & & & & & & & & & & 37.6 \\
\hline & & Mín. & & & & & & & & & & & & & & & & 26.6 \\
\hline & & Prom. $\left({ }^{\circ} \mathrm{C}\right)$ & & ת ת & 20 & 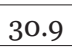 & & & & & & & & & & & & 31.5 \\
\hline \multirow{9}{*}{$\begin{array}{l}\text { हี } \\
\text { 壮 }\end{array}$} & \multirow{3}{*}{$\begin{array}{l}\text { Ancho } \\
16 \mathrm{~m}\end{array}$} & & & & & & & & & & & & & & & & & 37.6 \\
\hline & & Mín. & 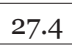 & 25.7 & 27.2 & 2 & o & 26.4 & 27 & 26 & .9 & 2 & 27.7 & 26.0 & 3 & 5 & 6 & 26.1 \\
\hline & & Prom & & & 80 & & & & & & & & & & & & & 30.8 \\
\hline & \multirow{3}{*}{$\begin{array}{l}\text { Ancho } \\
20 \mathrm{~m}\end{array}$} & & & & 39.0 & & & & & & & & & & & & & 8.4 \\
\hline & & Mín. $\left({ }^{\circ} \mathrm{C}\right)$ & & 26.0 & 27.4 & 25 . & 28.1 & 26.7 & 28. & 0 & 80 & 86 & 27.9 & 26.5 & & & 27.7 & 26.3 \\
\hline & & & & & & & & & & & & & & & & & & 31.4 \\
\hline & \multirow{3}{*}{$\begin{array}{l}\text { Ancho } \\
30 \mathrm{~m}\end{array}$} & Máx. & & 40.5 & 38.3 & 36 & 0.5 & 39.5 & 37 & 36 & 42.5 & 40.9 & 38.8 & 37.3 & . & - & 39.1 & 37.5 \\
\hline & & Mín. & 0.1 & 27. & 27 & 26 & & 27.8 & 28 & & & 27 & 28.0 & 26.7 & & & 28.1 & 27.0 \\
\hline & & Prom. $\left({ }^{\circ} \mathrm{C}\right)$ & 34.6 & 33.1 & 32.5 & 31.1 & 34.0 & 32.9 & 32.1 & 31.0 & 35.2 & 33.7 & 32.9 & 31.6 & 35.1 & 33.6 & 32.9 & 31.5 \\
\hline
\end{tabular}

$1^{*}$ "escenarios base", 2 * "escenarios forestados", 3 *escenarios con albedos optimizados", y 4 * "escenarios con estrategias combinadas".

consumo energético para el acondicionamiento térmico de edificios. Del análisis de los resultados se desprende que para:

- Escenarios base, etapa 1, las máximas diferencias térmicas entre los 24 escenarios alcanzan $3.8^{\circ} \mathrm{C}$ en la temperatura máxima, $0.6^{\circ} \mathrm{C}$ en la mínima y $1.4^{\circ} \mathrm{C}$ en la promedio. Esto se verifica para el escenario de $6 \mathrm{~m}$ de alto, $16 \mathrm{~m}$ de ancho y orientación NO-SE que muestra las menores temperaturas máxima y promedio $\left(39.1^{\circ} \mathrm{C}\right.$ y $\left.32.7^{\circ} \mathrm{C}\right)$, mientras que la menor temperatura mínima $\left(27.4^{\circ} \mathrm{C}\right)$ se registra en el escenario de $6 \mathrm{~m}$ de alto, $16 \mathrm{~m}$ de ancho y orientación N-S.

- Escenarios forestados, etapa 2, siempre se mantienen más frescos que los "escenarios base". Las máximas diferencias térmicas entre los 24 escenarios alcanzan $1.8^{\circ} \mathrm{C}$ en la temperatura máxima, $0.7^{\circ} \mathrm{C}$ en la mínima y $0.9^{\circ} \mathrm{C}$ en la promedio. Nuevamente el escenario de $6 \mathrm{~m}$ de alto, $16 \mathrm{~m}$ de ancho y orientación NO-SE presenta las menores temperaturas máxima y promedio $\left(37.9^{\circ} \mathrm{C}\right.$ y $\left.31.3^{\circ} \mathrm{C}\right)$, mientras que la menor temperatura mínima $\left(25.3^{\circ} \mathrm{C}\right)$ se registra en el escenarios de $3 \mathrm{~m}$ de alto, $16 \mathrm{~m}$ de ancho y orientación N-S.

- Escenarios con albedos optimizados, etapa 3, se verifica que siempre se mantienen más frescos que los "escenarios base". Las máximas diferencias térmicas entre los 24 escenarios alcanzan $2.7^{\circ} \mathrm{C}$ en la temperatura máxima, $0.7^{\circ} \mathrm{C}$ en la mínima y $1.2^{\circ} \mathrm{C}$ en la promedio. El escenario de $6 \mathrm{~m}$ de alto, $30 \mathrm{~m}$ de ancho y orientación NO-SE muestra las menores temperaturas máxima y promedio $\left(37.2^{\circ} \mathrm{C}\right.$ y $\left.32.1^{\circ} \mathrm{C}\right)$, mientras que la menor temperatura mínima $\left(27.2^{\circ} \mathrm{C}\right)$ se registra en el escenario de $6 \mathrm{~m}$ de alto, $16 \mathrm{~m}$ de ancho y orientación N-S.

- Escenarios con estrategias combinadas, etapa 4, siempre se mantienen más frescos que los "escenarios base". Las máximas diferencias térmicas entre los 24 escenarios alcanzan $1.3^{\circ} \mathrm{C}$ en la temperatura máxima, $0.8^{\circ} \mathrm{C}$ en la mínima y $0.8^{\circ} \mathrm{C}$ en la promedio. El escenario de $6 \mathrm{~m}$ de alto, $30 \mathrm{~m}$ de ancho y orientación NO-SE muestra la menor temperatura máxima $\left(36.2^{\circ} \mathrm{C}\right)$. El de $6 \mathrm{~m}$ de alto, $16 \mathrm{~m}$ de ancho y orientación N-S la menor temperatura promedio $\left(30.5^{\circ} \mathrm{C}\right)$; y el de $3 \mathrm{~m}$ de alto, $16 \mathrm{~m}$ de ancho y orientación N-S la menor temperatura mínima $\left(25 \cdot 1^{\circ} \mathrm{C}\right)$.
Es decir que a priori, sin considerar que estrategia de rehabilitación se utilice (forestación o albedo), las orientaciones de calle más frescas son la NO-SE y N-S. Siendo estos resultados coincidentes con los de Krüger, et al. (58) y Ali-Toudert \& Mayer (59). Asimismo, al aplicar las estrategias de mejora en forma individual o conjunta, se verifica que para la orientación NO-SE los impactos son menores, ya que la condición de base es la más fresca. La tabla 5 muestra un esquema de

Tabla 5. Escenarios más frescos para cada etapa.

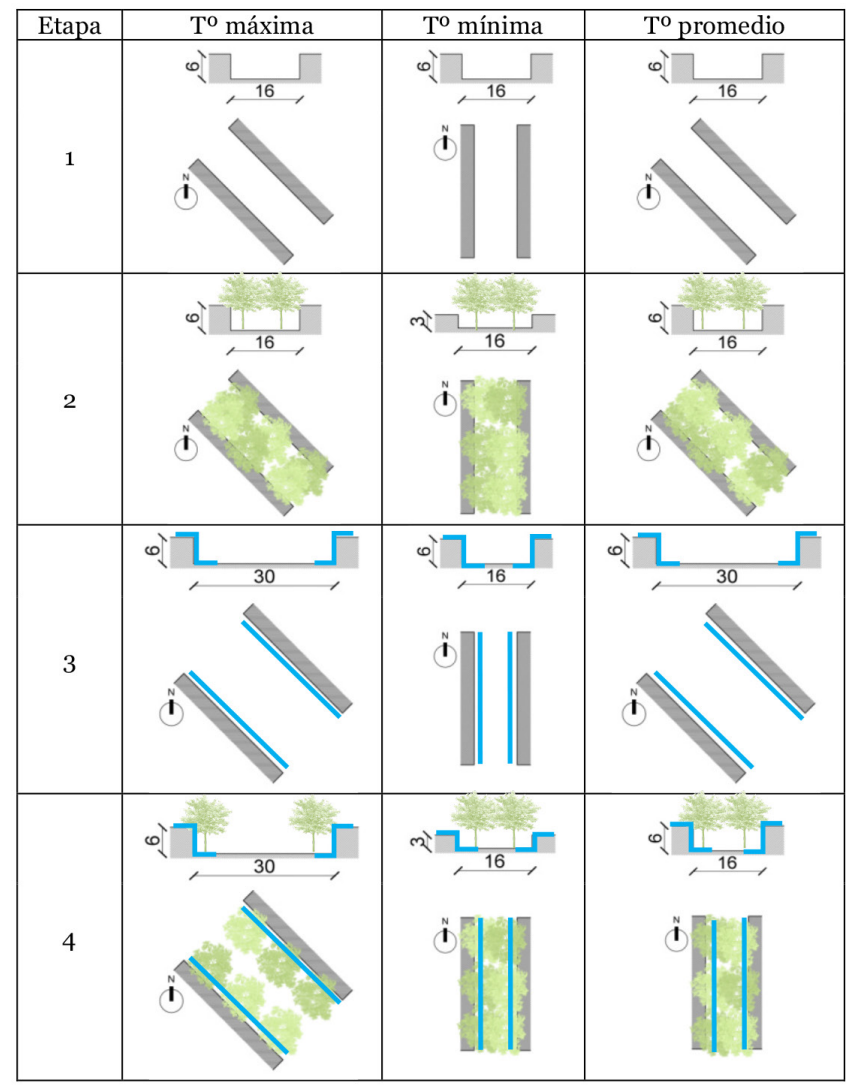


los perfiles y planimetrías de los escenarios más frescos para cada etapa, diferenciados según temperaturas máxima, mínima y promedio.

Las Fig. 3 y 4 muestran las diferencias térmicas alcanzadas entre los escenarios base (etapa 1) y los escenarios con las estrategias incorporadas (etapas 2, 3 y 4). Se agrupan los comportamientos según temperaturas máximas (color rojo), mínimas (color azul) y promedios (color verde) para cada ancho de calle, diferenciados para alturas de edificación de 3 y $6 \mathrm{~m}$. Cuando se analizan las reducciones de temperatura, se detecta de manera clara que las mayores diferencias se encuentran al aplicar las estrategias combinadas. El análisis de los efectos potenciales de enfriamiento se analizan de acuerdo a:

- Temperaturas máximas: el escenario que combina todas las estrategias (etapa 4) muestra ser el más eficaz -mayor disminución de la temperatura máxima- en todas las orientaciones. De modo particular, en aquellos casos cuya

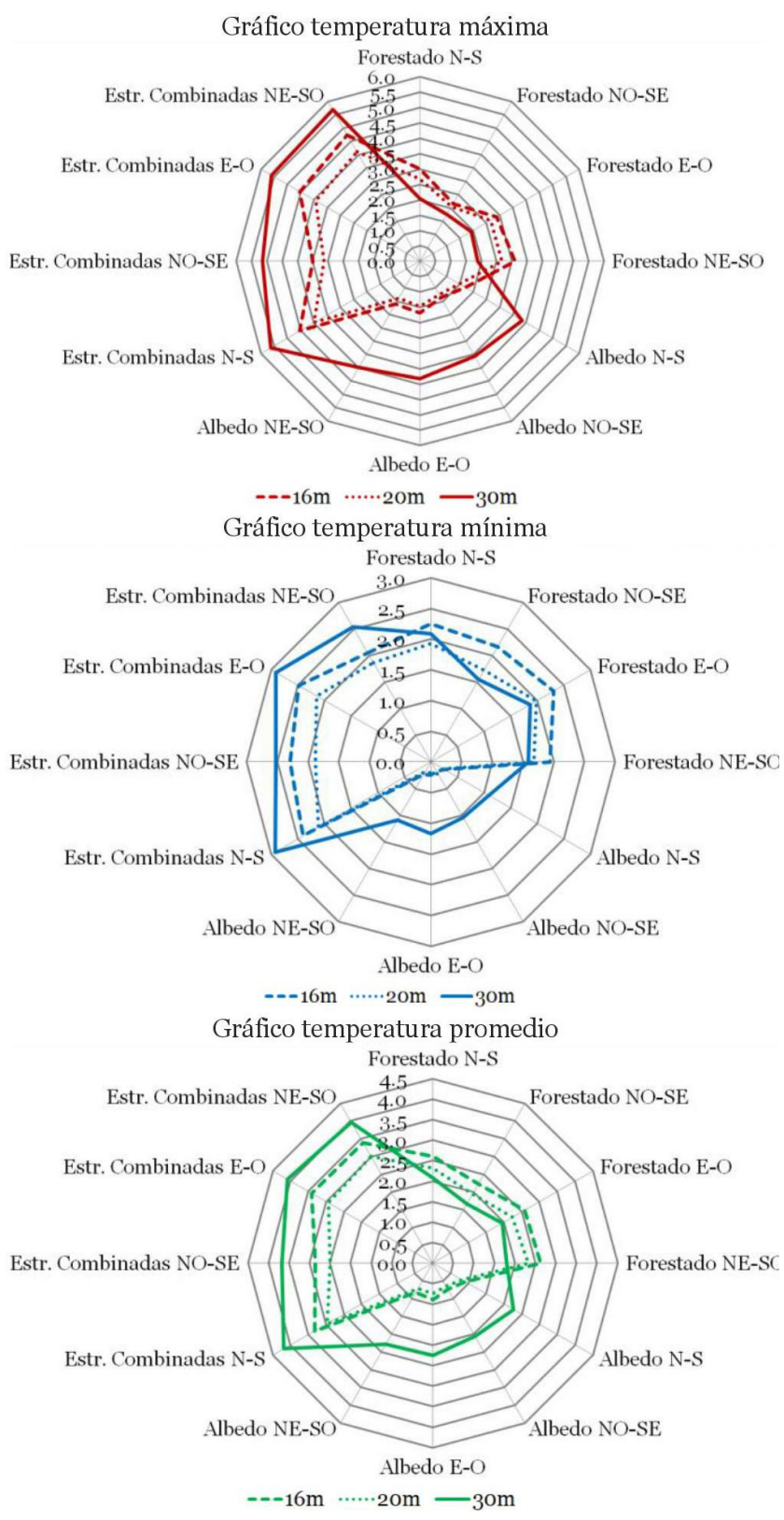

Figura 3. Diferencias térmicas escenarios de $3 \mathrm{~m}$ de altura. orientación resulta ser la más cálida (NE-SO) con ancho de calle de $30 \mathrm{~m}$ y altura de $3 \mathrm{~m}$, la disminución de temperatura máxima alcanza los $5 \cdot 7^{\circ} \mathrm{C}$.

- Temperaturas mínimas: las mayores disminuciones se registran a partir de aplicar todas las estrategias combinadas o sólo forestar las calles (etapas 2 y 4). Poniendo en evidencia, que la modificación de albedos no tiene impacto significativo sobre la condición térmica nocturna. Al igual que en el punto anterior, las mayores disminuciones de temperatura mínima $\left(2.9^{\circ} \mathrm{C}\right)$ se consiguen en las calles de $30 \mathrm{~m}$ de ancho, en aquellos casos cuya orientación resulta ser la más fresca (N-S) y con alturas de $3 \mathrm{~m}$.

- Temperaturas promedio: las estrategias más eficaces son la forestación y la combinación de estrategias (etapas 2 y 4); mientras que el albedo colabora en menor medida en la reducción de las temperaturas. La incidencia en la disminución de la temperatura más importante se genera en calles de $30 \mathrm{~m}$ de ancho, orientados $\mathrm{N}-\mathrm{S}$ con alturas de $3 \mathrm{~m}$ $\left(4 \cdot 2^{\circ} \mathrm{C}\right)$.

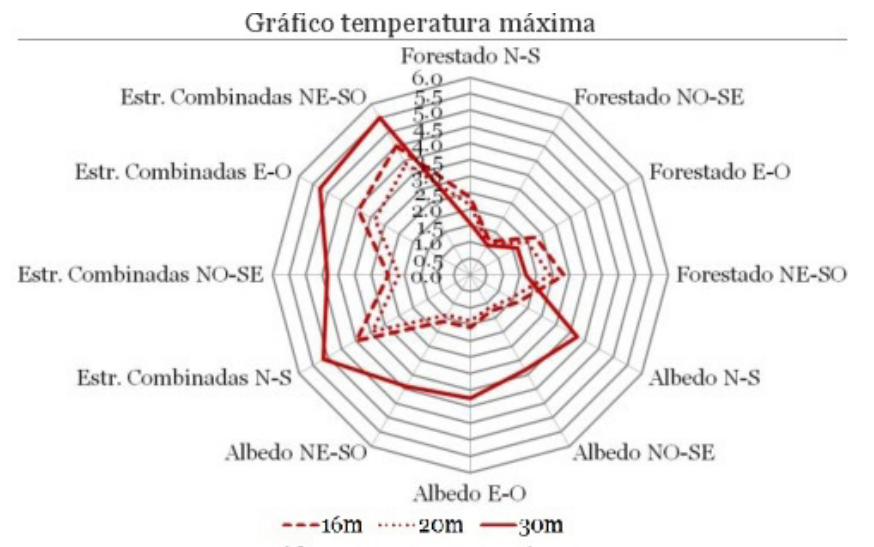

Gráfico temperatura mínima

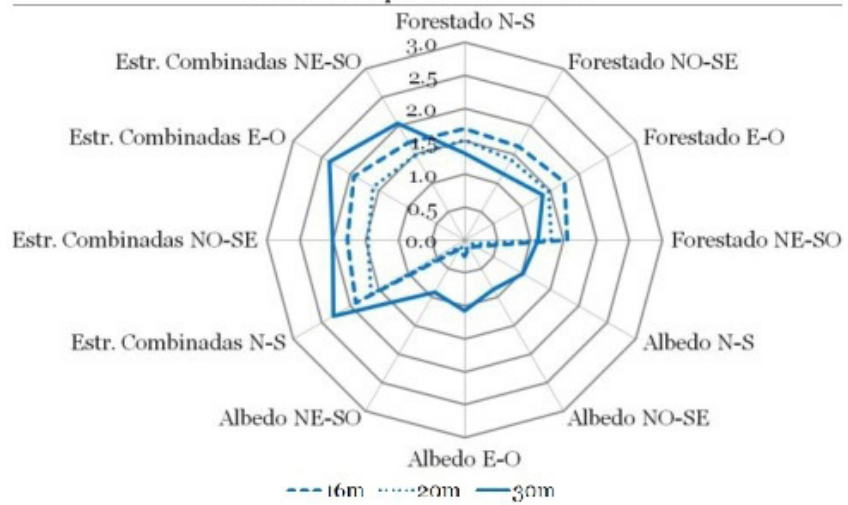

Gráfico temperatura promedio Forestado N-S

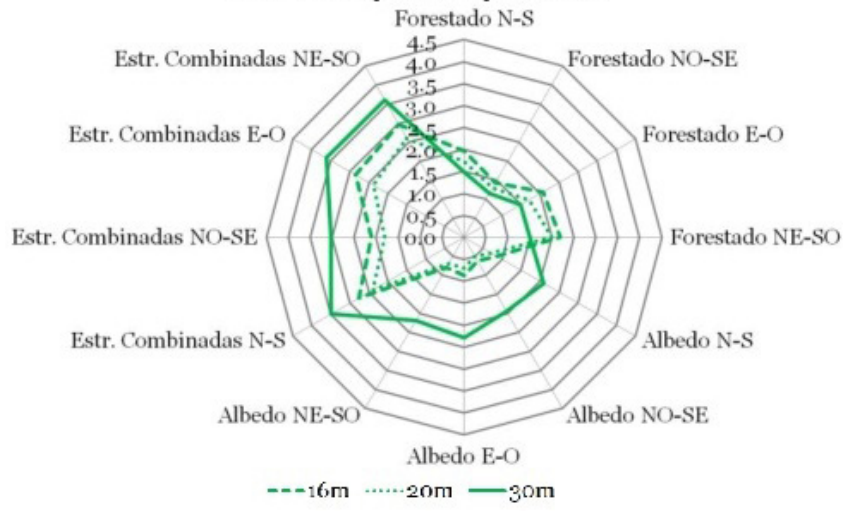

Figura 4. Diferencias térmicas escenarios de $6 \mathrm{~m}$ de altura. 


\subsection{Nivel de eficacia de las estrategias de reducción del calentamiento urbano}

La tabla 6 muestra el nivel de eficacia de las dos estrategias de disminución de temperaturas, y la combinación de ambas, que fueron evaluadas. La eficacia de cada estrategia se determinó como la relación entre el resultado alcanzado (RA) sobre el resultado esperado (RE). Donde el RE es el comportamiento del escenario base (etapa 1) para cada ancho de calle y altura de edificación; y el RA es el valor más fresco obtenido en cada una de las tres etapas de simulación con las estrategias aplicadas. $\mathrm{Al}$ analizar la tabla se visualiza que la estrategia más eficaz es la incorporación de forestación de alineación en los canales viales junto con la optimización de albedos de los materiales expuestos (etapa 4). Alcanzando los mayores porcentajes de mejora en hasta un $12 \%$ en la disminución de la temperatura máxima, 10\% en la mínima y $11 \%$ en la promedio. En segundo lugar se vuelve a corroborar que la incorporación de forestación (etapa 2) muestra un buen desempeño; alcanzando reducciones de hasta $5 \%$ en la temperatura máxima, $8 \%$ en la mínima y $7 \%$ en la promedio. En tercer lugar, la optimización de albedos (etapa 3) reduce la temperatura máxima en hasta un $9 \%$, un $4 \%$ mínima y $6 \%$ en la promedio; pero esta estrategia demuestra su eficacia en los canales más expuestos (ancho de calle $30 \mathrm{~m}$ ). Es decir, que si se debe priorizar solo una estrategia de mejora, se recomienda incluir forestales de alineación que sombreen las superficies expuestas ya que alcanzan reducciones térmicas en los 3 anchos de calle. Otra característica en cuanto a la forma del canal vial a destacar, es que la eficacia de las estrategias es mayor en las alturas de edificación de $3 \mathrm{~m}$.

\subsection{Contraste de comportamiento entre escenarios "base" versus "estrategias combinadas"}

Se presenta a continuación la Fig. 5 que muestra en términos porcentuales el comportamiento térmico de los 24 escenarios base y los 24 escenarios con estrategias combinadas, diferenciados según temperaturas del aire máxima, mínima y promedio. El objetivo de esta contrastación es el de definir la capacidad de mejora térmica de cada escenario. La figura muestra en la columna de la izquierda un gradiente de colores que referencia los rangos de temperatura. Cabe aclarar que los mayores rangos de temperaturas máximas y promedios tienen los colores más intensos (más cálido), y que en las temperaturas mínimas esta referencia se invierte y los colores más intensos se asocian a menores temperaturas (más frescos). Al analizar la Fig. 5 se visualiza que:

- Escenarios base, el 50\% de los escenarios alcanzan una temperatura máxima en el rango entre 41.9 a $42.9^{\circ} \mathrm{C}$, el 42\% alcanzan una temperatura mínima en el rango mayor a $27.9^{\circ} \mathrm{C}$ y el $50 \%$ alcanzan una temperatura promedio en el rango entre 33.9 a $34.9^{\circ} \mathrm{C}$.

- Escenarios con estrategias combinadas, el 67\% de los escenarios alcanzan una temperatura máxima en el rango entre 36.9 a $37.9^{\circ} \mathrm{C}$, el $58 \%$ alcanzan una temperatura mínima en el rango entre 25.9 a $26.9^{\circ} \mathrm{C}$ y el $75 \%$ alcanzan una temperatura promedio en el rango entre 30.9 a $31.9^{\circ} \mathrm{C}$.

Es decir que la capacidad de mejora al incorporar ambas estrategias se visualiza en la disminución de la temperatura máxima de 5 a $6^{\circ} \mathrm{C}$ en el $67 \%$ de los casos, del orden de 2 a $3^{\circ} \mathrm{C}$ en la temperatura mínima en el $58 \%$ de los casos y del orden de 3 a $4^{\circ} \mathrm{C}$ en la temperatura promedio en el $75 \%$ de los casos. Siendo estos hallazgos similares a los obtenidos por Alchapar et al. (60), donde en la ciudad de Campinas - Brasil la temperatura máxima del aire se redujo en hasta $4^{\circ} \mathrm{C}$ en un entorno de baja densidad y $5{ }^{\circ} \mathrm{C}$ en alta densidad.

\section{CONCLUSIONES}

Los resultados de este trabajo indican que existen diferencias en el comportamiento térmico de los 96 escenarios urbanos simulados que se diferencian según altura de edificación, ancho y orientación de calle. A través de la incorporación de dos estrategias de reducción del calentamiento urbano y la combinación de ambas - forestación y albedos optimizados- se determinó el nivel de eficacia para reducir el calentamiento urbano.

Tabla 6. Nivel de eficacia de las estrategias.

\begin{tabular}{|c|c|c|c|c|c|c|c|c|c|}
\hline & $\begin{array}{c}\text { Ancho } \\
\text { (m) }\end{array}$ & Temp. $\left({ }^{\circ} \mathrm{C}\right)$ & $\begin{array}{c}\text { Etapa } 2 \\
\text { (RA) }\end{array}$ & $\begin{array}{l}\text { Dif } \\
(\%)\end{array}$ & $\begin{array}{c}\text { Etapa } 3 \\
\text { (RA) }\end{array}$ & $\begin{array}{l}\text { Dif } \\
(\%)\end{array}$ & $\begin{array}{c}\text { Etapa } 4 \\
+ \text { eficaz (RA) }\end{array}$ & Dif (\%) & $\begin{array}{c}\text { Etapa } 1 \\
\text { - eficaz } \\
\text { (RE) }\end{array}$ \\
\hline \multirow{9}{*}{ 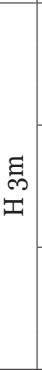 } & \multirow{3}{*}{16} & Máx. & 38.5 & 5 & 39.3 & 3 & 37.2 & 9 & 40.7 \\
\hline & & Mín. & $25 \cdot 3$ & 8 & 27.3 & 1 & 25.1 & 9 & 27.6 \\
\hline & & Prom. & 31.3 & 7 & 32.8 & 2 & 30.6 & 9 & 33.5 \\
\hline & \multirow{3}{*}{20} & Máx. & 38.8 & 5 & 39.6 & 3 & 37.7 & 8 & 40.9 \\
\hline & & Mín. & $25 \cdot 7$ & 7 & 27.4 & 1 & $25 \cdot 5$ & 8 & 27.6 \\
\hline & & Prom. & 31.7 & 6 & 33.0 & 2 & 31.1 & 8 & 33.6 \\
\hline & \multirow{3}{*}{30} & Máx. & 40.0 & 4 & 38.2 & 9 & 36.6 & 12 & 41.8 \\
\hline & & Mín. & 26.9 & 7 & 27.9 & 4 & 26.1 & 10 & 29.0 \\
\hline & & Prom. & 33.0 & 5 & 32.7 & 6 & 30.9 & 11 & 34.8 \\
\hline \multirow{9}{*}{$\begin{array}{l}\text { घี } \\
\text { 王 }\end{array}$} & \multirow{3}{*}{16} & Máx. & 37.9 & 3 & 37.8 & 3 & 36.6 & 6 & 39.1 \\
\hline & & Mín. & $25 \cdot 7$ & 6 & 27.2 & 1 & $25 \cdot 5$ & 7 & 27.4 \\
\hline & & Prom. & 31.3 & 4 & 32.1 & 2 & 30.5 & 7 & 32.7 \\
\hline & \multirow{3}{*}{20} & Máx. & 38.3 & 3 & 38.2 & 3 & 37.2 & 5 & 39.4 \\
\hline & & Mín. & 26.0 & 6 & 27.4 & 0 & 25.9 & 6 & 27.5 \\
\hline & & Prom. & 31.6 & 4 & 32.4 & 2 & 31.0 & 6 & 32.9 \\
\hline & \multirow{3}{*}{30} & Máx. & 39.5 & 3 & 37.2 & 8 & 36.2 & 11 & 40.5 \\
\hline & & Mín. & 27.4 & 5 & 27.7 & 4 & 26.4 & 8 & 28.7 \\
\hline & & Prom. & 32.9 & 3 & 32.1 & 6 & 31.0 & 9 & 34.0 \\
\hline
\end{tabular}




\begin{tabular}{c} 
Temperatura máxima \\
\hline$\geq 36.9$ \\
$\geq 36.9<37.9$ \\
$\geq 37.9<38.9$ \\
$\geq 38.9<39.9$ \\
$\geq 39.9<40.9$ \\
$\geq 40.9<41.9$ \\
$\geq 41.9<42.9$ \\
$\geq 42.9<43.9$ \\
Temperatura mínima
\end{tabular}

$$
\begin{gathered}
\geq 25.9 \\
\geq 25.9<26.9 \\
\geq 26.9<27.9 \\
\geq 27.9<28.9 \\
\geq 28.9<29.9
\end{gathered}
$$

Temperatura promedio
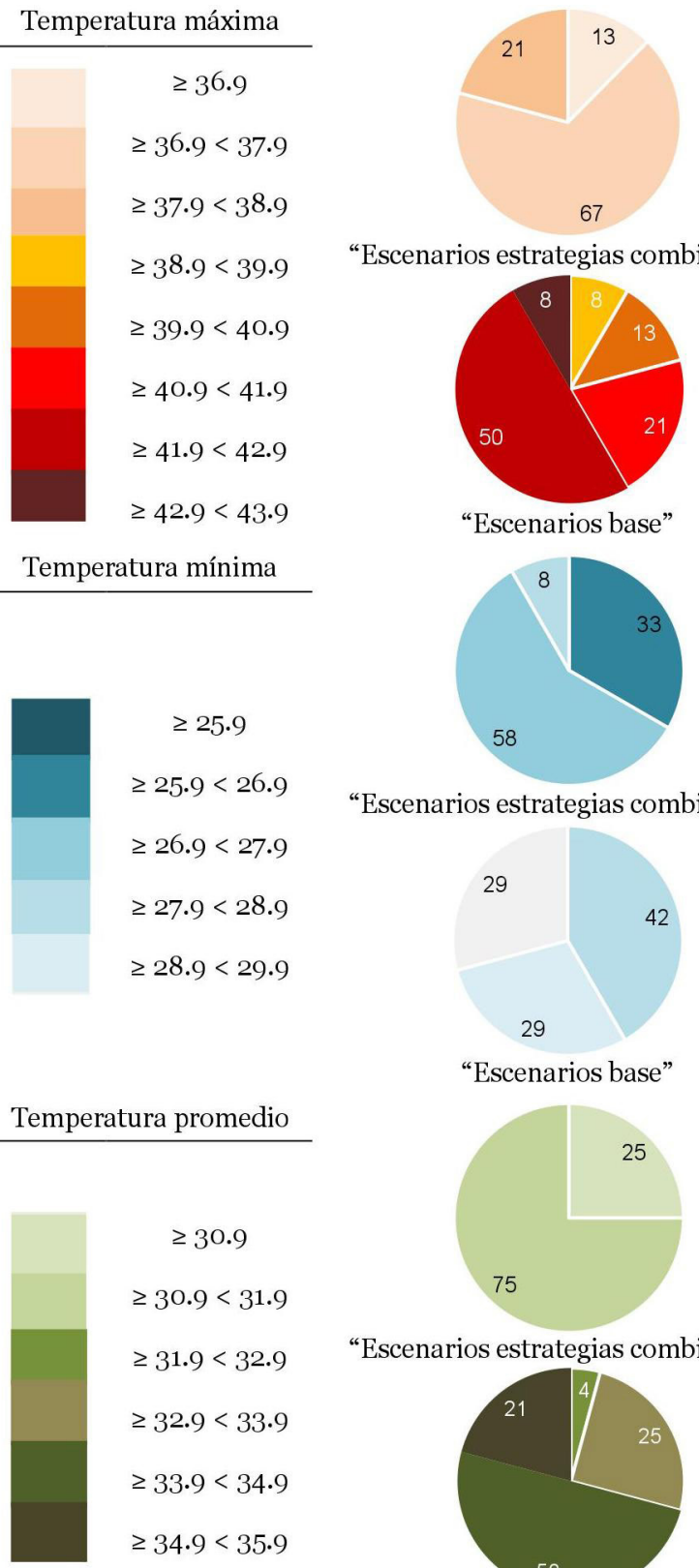

"Escenarios estrategias combinadas"
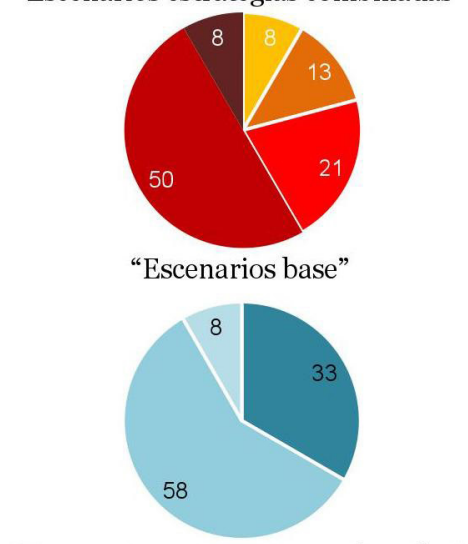

"Escenarios estrategias combinadas"

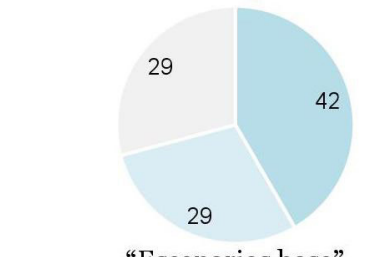

"Escenarios base"

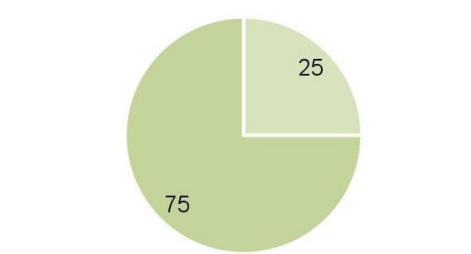

"Escenarios estrategias combinadas"

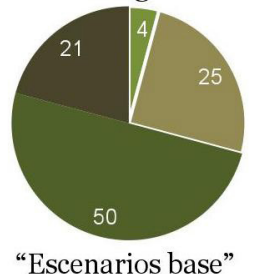

Figura 5. Contraste comportamiento escenarios, etapas 1 y 4.

La etapa 1, "escenarios base” (evaluación morfológica), determinó que las alturas de edificación de $6 \mathrm{~m}$ tienen mejores desempeños térmicos durante el día, y las de $3 \mathrm{~m}$ durante el periodo nocturno. Con respecto a los anchos de calle, los escenarios de $30 \mathrm{~m}$ tienen los peores desempeños térmicos (mayor exposición a la radiación solar); siendo los mejores escenarios los anchos de 16 y 20 m. En cuanto a la orientación los sentidos N-S y NO-SE son los que muestran los mejores desempeños. La inclusión de forestación de alineación en la etapa de simulación 2, "escenarios forestados", mejora las condiciones de los "escenarios base" demostrando ser más eficaz en la reducción de temperaturas en hasta un 5\% en la máxima, $8 \%$ en la mínima y $7 \%$ en la promedio. La adecuada selección de materiales con valores de albedos optimizados, etapa 3 "escenarios con albedos optimizados", resulta más eficaz para disminuir las temperaturas de los canales más expuestos (anchos de 30m); hasta un $9 \%$ en la máxima, un $4 \%$ mínima y $6 \%$ en la promedio. Esto demuestra que si se debe priorizar solo una estrategia de mejora, se recomienda incluir forestales de alineación que sombreen las superficies expuestas ya que mejora la respuesta térmica para los 3 anchos de calle. Por último, la etapa 4 "escenarios con estrategias combinadas", resulta ser la más eficaz ya que se potencian los beneficios individuales de cada estrategia; alcanzando disminuciones de hasta un $12 \%$ en la temperatura máxima, $10 \%$ en la mínima y $11 \%$ en la promedio. Siendo, los escenarios urbanos con ancho de calle de $16 \mathrm{~m}$ y orientaciones N-S y NO-SE, los que registran las mayores mejoras térmicas al aplicar las estrategias combinadas (comportamientos entre 36.6 a $37.5^{\circ} \mathrm{C}$ en la temperatura máxima, $25.1 \mathrm{a} 26.3^{\circ} \mathrm{C}$ en la mínima y 30.5 a $30.7^{\circ} \mathrm{C}$ en la promedio).

Estos hallazgos ponen en relevancia el impacto térmico que generan las decisiones planificación y diseño de las áreas urbanas; como así también el nivel de eficacia de las diversas estrategias asociadas a la morfología. La introducción de la evaluación y diagnóstico microclimático como herramientas para definir estrategias direccionadas a mejorar las condiciones térmicas exteriores contribuye a disminuir los efectos de la urbanización sobre el clima, la calidad de vida de las personas, el consumo de energía y la salud.

\section{LIMITACIÓN DE ESTE ESTUDIO}

El modelo de sensor utilizado en este estudio es recomendado para uso interior, siendo el abrigo meteorológico el diferenciante con su versión outdoor PRO. Es por ello, que para proteger al sensor con el que tomaron las mediciones in situ se diseñó y fabricó un abrigo con materiales accesibles y disponibles en el ámbito local. Este abrigo (caja de PVC perforada) considera las recomendaciones de Terando et al. (61), Huwald et al. (62) y Richardson et al. (63), que mencionan que se deberá garantizar: (i) el flujo libre de aire alrededor del sensor, (ii) la mínima exposición del sensor a la radiación solar, y (iii) la mínima absorción de radiación por el abrigo. Siendo esta condición viable para obtener los datos microclimáticos necesarios para validar el modelo físico a través de un minucioso ajuste (tabla 2). Por último se destaca que estos datos fueron utilizados para contrastar y analizar diferencias térmicas entre los 96 escenarios, no entre diversos puntos de medición. 


\section{REFERENCIAS}

(1) Rogers, R., y Gumuchdjian, P. (2000). Ciudades para un pequeño planeta. Barcelona: GG.

(2) Higueras, E. (2009). La ordenanza bioclimática de Tres Cantos, Madrid. Últimos avances en planificación ambiental y sostenible. Revista de Urbanismo, 20. Recuperado de https://revistaurbanismo.uchile.cl/index.php/RU/article/view/8

(3) UN-HABITAT. (2016). World Cities Report. United Nations Human Settlements Programme.

(4) Font, P. (1998). Ordenación y Planificación Territorial. Madrid: Síntesis.

(5) Tumini, I. (2012). El microclima urbano de los espacios abiertos. Estudio de casos en Madrid (Tesis doctoral). Universidad Politécnica de Madrid.

(6) IPCC. (2014). Climate Change 2014. Synthesis Report Summary for Policymakers. Retrieved from https://www.ipcc. ch/pdf/assessment-report/ar5/syr/AR5_SYR_FINAL_SPM.pdf

(7) Oke, T. (1982). The energetic basis of the urban heat island. Quarterly Journal Royal Meteorological Society, 108(455): 1-24. https://doi.org/10.1002/qj.49710845502

(8) Givoni, B. (1998). Climate consideration in buildings and urban design. United States of America: Van Nostrand Reinhold.

(9) Ratti, C., Raydan, D., y Steemers, K. (2003). Building form and environmental performance: archetypes, analysis and an arid climate. Energy and Buildings, 35(1): 49-59. https://doi.org/10.1016/So378-7788(02)00079-8

(10) Scudo G., y Ochoa de la Torre, J. M. (2003). Spazi verdi urbani, la vegetazione come strumento di progetto per il comfort ambientale negli spazi abitati. Napoli: Esselibri.

(11) ICLEI. (1994). Carta de las ciudades europeas hacia la sostenibilidad. En Conferencia europea sobre ciudades sostenibles, Aalborg - Dinamarca, 24 al 27 de mayo.

(12) Eliasson, I. (2000). The use of climate knowledge in urban planning. Landscape and Urban Planning, 48(1-2): 31-44. https://doi.org/10.1016/So169-2046(oo)00034-7

(13) Svensson, M. y Eliasson, I. (2002). Diurnal air temperatures in built-up areas in relation to urban planning. Landscape and Urban Planning, 61(1): 37-54. https://doi.org/10.1016/So169-2046(02)ooo76-2

(14) Alcoforado, M., Andrade, H., Lopes A. y Vasconcelos, J. (2009). Application of climatic guidelines to urban planning: the example of Lisbon (Portugal). Landscape and Urban Planning, 90(1-2): 56-65. https://doi.org/10.1016/j.landurbplan.2008.10.006

(15) Ren, C., Ng, E. y Katzschner, L. (2011). Urban climatic map studies: a review. International Journal of Climatology, 31(15): 2213-2233. https://doi.org/10.1002/joc.2237

(16) Grimmond, C., Roth, M., Oke, T., Au, Y., Best, M., Betts, R., Carmichael G, Cleugh, H., Dabberdt, W., Emmanuel, R., Freitas, E., Fortuniak, K., Hanna, S., Klein, P., Kalkstein, L., Liu, C., Nickson, A., Pearlmutter, D., Sailor, D. y Voogt, J. (2010). Climate and more sustainable cities: climate information for improved planning and management of cities (producers/ capabilities perspective). Procedia Environmental Sciences, 1:247-274. https://doi.org/10.1016/j.proenv.2010.09.016

(17) Ng, E. (2012). Towards planning and practical understanding of the need for meteorological and climatic information in the design of high-density cities: a case-based study of Hong Kong. International Journal of Climatology, 32(4): 582598. https://doi.org/10.1002/joc.2292

(18) Norton, B., Coutts, A., Livesley, S., Harris, R., Hunter, A. y Williams, N. (2015). Planning for cooler cities: A framework to prioritise green infrastructure to mitigate high temperatures in urban landscapes. Landscape and Urban Planning, 134: 127-138. https://doi.org/10.1016/j.landurbplan.2014.10.018

(19) Grimmond, C., y Oke, T. (1999). Aerodynamic properties of urban areas derived from analysis of urban form. Journal of Applied Meteorology, 38(9): 1262-1292. https://doi.org/10.1175/1520-0450(1999)038<1262:APOUAD>2.0.CO;2

(20) Shashua-Bar, L., Tzamir, Y., y Hoffman, M. (2004). Thermal effects of building geometry and spacing on the urban canopy layer microclimate in a hot-humid climate summer. International Journal of Climatology, 24(13): 1729-1742. https://doi.org/10.1002/joc.1092

(21) Arnfield, A. (2013). Canyon geometry, the urban fabric and nocturnal cooling: a simulation approach. Physical Geography, 11(3): 220-239. https://doi.org/10.1080/02723646.1990.10642404

(22) Cabras, E. (2014). Efectos de la morfología de las calles en el fenómeno de la isla de calor urbana en la ciudad de Barcelona (Tesis doctoral). Universidad Politécnica de Cataluña.

(23) Santamouris, M., Ding, L., Fiorito, F., Oldfield, P., Osmond, P., Paolini, R., Synnefa, A., y Prasad, D., (2017). Passive and active cooling for the outdoor built environment - Analysis and assessment of the cooling potential of mitigation technologies using performance data from 220 large scale projects. Solar Energy, 154: 14-33. https://doi.org/10.1016/j. solener.2016.12.006

(24) Rizwan, A., Dennis, L. \& Liu, C. (2008). A review on the generation, determination and mitigation of Urban Heat Island. Journal of Environmental Sciences, 20(1): 120-128. https://doi.org/10.1016/S1001-0742(08)60019-4

(25) Kleerekoper, L., Van Esch, M., y Salcedo, T., (2012). How to make a city climate-proof, addressing the urban heat island effect. Resources, Conservation and Recycling, 64: 30-38. https://doi.org/10.1016/j.resconrec.2011.06.004

(26) Jusuf, S.K., Wong, N.H. \& Hagen, E. (2007). The influence of land use on the urban heat island in Singapore. Habitat Internacional, 31(2): 232-242. https://doi.org/10.1016/j.habitatint.2007.02.006

(27) NRDC, Raimi + Associates and the Natural Resources Defense Council (2011). A Citizen's Guide to LEED for Neighborhood Development: How to Tell if Development is Smart and Green.

(28) Ruiz, M. A., Sosa, M. B., Correa, E., \& Cantón, M. A. (2017). Design tool to improve daytime thermal comfort and nighttime cooling of urban canyons. Urban Planning and Landscape, 167: 249-256. https://doi.org/10.1016/j.landurbplan.2017.07.002 
(29) Sanusi, R., Johnstone, D., May, P., \& Livesley, S. (2016). Street orientation and side of the street greatly influence the microclimatic benefits street trees can provide in summer. Journal of Environmental Quality, 45(1): 167-174. https:// doi.org/10.2134/jeq2015.01.0039

(30) Bakarmana, M. \& Changa, J. (2015). The influence of height/width ratio on urban heat island in hot-arid climates. Procedia Engineering, 118: 101-108. https://doi.org/10.1016/j.proeng.2015.08.408

(31) Bourbia F. y Awbi, H. (2004a). Building cluster and shading in urban canyon for hot dry climate: Part 1: Air and surface temperature measurements. Renewable Energy, 29(2): 249-262. https://doi.org/10.1016/So960-1481(03)o0170-8

(32) Bourbia F. y Awbi, H. (2004b). Building cluster and shading in urban canyon for hot dry climate: part 2: shading simulations. Renewable Energy, 29(2): 291-301. https://doi.org/10.1016/So960-1481(03)o0171-X

(33) Middel, A., Häb, K., Brazel, A. J., Martin, C. A., \& Guhathakurta, S. (2014). Impact of urban form and design on midafternoon microclimate in Phoenix Local Climate Zones. Landscape and Urban Planning, 122: 16-28. https://doi. org/10.1016/j.landurbplan.2013.11.004

(34) Middel, A., Chhetri, N., \& Quay, R. (2015). Urban forestry and cool roofs: Assessment of heat mitigation strategies in Phoenix residential neighborhoods. Urban Forestry \& Urban Greening, 14(1): 178-186. https://doi.org/10.1016/j. ufug.2014.09.010

(35) Schmidt, M. (2006). The contribution of rainwater harvesting against global warming. Technische Universität Berlin, IWA Publishing.

(36) Kravcík, M., Pokorny, Kohutiar, J., Kovác, M. y Tóth, E., (2007). Water for the Recovery of the Climate - A New Water Paradigm. Building Environment, 45: 192-201.

(37) Nishimura, N., Nomura, T., Iyota, H. y Kimoto, S., (1998). Novel water facilities for creation of comfortable urban micrometeorology. Solar Energy, 64(4-6): 197-207. https://doi.org/10.1016/Soo38-092X(98)o0116-9

(38) Barakat, A., Ayad, H. \& El-Sayed, Z. (2017). Urban design in favor of human thermal comfort for hot arid climate using advanced simulation methods. Alexandria Engineering Journal, 56(4): 533-543. https://doi.org/10.1016/j. aej.2017.04.008

(39) Todhunter, P. (2013). Microclimatic variations attributable to urban-canyon asymmetry and orientation. Physical Geography, 11(2): 131-141. https://doi.org/10.1080/02723646.1990.10642398

(40) Mirzaei, P., y Haghighat, F., (2010). Approaches to study Urban Heat Island e Abilities and limitations. Building and Environment, 45(10): 2192-2201. https://doi.org/10.1016/j.buildenv.2010.04.001

(41) Casey, M., y Wintergerst, T. (2000). Quality and trust in industrial CFD - best practice guidelines. ERCOFTAC Special Interest Group, Sulzer Innotec, Fluid Dynamics Laboratory.

(42) van Hooff, T., y Blocken, B., (2010). Coupled urban wind flow and indoor natural ventilation modelling on a high-resolution grid: a case study for the Amsterdam Arena stadium. Environmental Modelling \& Software, 25(1): 51-65. https:// doi.org/10.1016/j.envsoft.2009.07.008

(43) Arnfield, A. (2013). Canyon geometry, the urban fabric and nocturnal cooling: a simulation approach. Physical Geography, 11(3): 220-239. https://doi.org/10.1080/02723646.1990.10642404

(44) Blocken, B. (2015). Computational Fluid Dynamics for urban physics: importance, scales, possibilities, limitations and ten tips and tricks towards accurate and reliable simulations. Building Environment, 91: 219-45. https://doi. org/10.1016/j.buildenv.2015.02.015

(45) Allegrini, J., Dorer, V., y Carmeliet, J., (2015). Influence of morphologies on the microclimate in urban neighbourhoods. Journal of Wind Engineering and Industrial Aerodynamics, 144: 108-117. https://doi.org/10.1016/j.jweia.2015.03.024

(46) Stocco, S., Cantón, A. \& Correa, E. (2018). Alternativas de diseño para mejorar el desempeño ambiental de plazas urbanas de Mendoza (Argentina). Evaluación mediante simulación con ENVI-met 3.1. Informes de la Construcción, 70(550), e253. https://doi.org/10.3989/ic.16.154

(47) Acero, J. \& Arrizabalaga, J. (2018). Evaluating the performance of ENVI-met model in diurnal cycles for different meteorological conditions. J. Theor Appl Climatol, 131: 455-469. https://doi.org/10.1007/so0704-016-1971-y

(48) López-Cabeza, V.P., Galán-Marín, C., Rivera-Gómez, C. \& Roa-Fernández, J. (2018). Courtyard microclimate ENVI-met outputs deviation from the experimental data. Building and Environment, 144: 129-141. https://doi.org/10.1016/j.buildenv.2018.08.013

(49) Salata, F.,Golasi, I., de Lieto Vollaro, R. \& de Lieto Vollaro, A. (2016). Urban microclimate and outdoor thermal comfort. A proper procedure to fit ENVI-met simulation outputs to experimental data. Sustainable Cities and Society, 26: 318343. https://doi.org/10.1016/j.scs.2016.07.005

(50) Tsoka, S., Tsikaloudaki, A. \& Theodosiou, T. (2018). Analyzing the ENVI-met microclimate model's performance and assessing cool materials and urban vegetation applications-A review. Sustainable Cities and Society, 43: 55-76. https:// doi.org/10.1016/j.scs.2018.08.009

(51) Bórmida, E., (1986). Mendoza, modelo de ciudad oasis. Revista SUMMA, 226: 68-72.

(52) IPV. (2010). Instituto Provincial de la Vivienda, Mendoza.

(53) INDEC. (2010). Censo nacional de población, hogares y viviendas.

(54) Oke, T. (2004). Initial guidance to obtain representative meteorological observations at urban sites. IOM Report No. 81, World Meteorological Organization. Retrevied from https://www.wmo.int/pages/prog/www/IMOP/publications/ IOM-81/IOM-81-UrbanMetObs.pdf

(55) Correa, E. (2006). Isla de Calor Urbana. El caso del área metropolitana de Mendoza (Tesis doctoral). Universidad Nacional de Salta.

(56) Wang, Y. \& Akbari, H. (2016). Analysis of urban heat island phenomenon and mitigation solutions evaluation for Montreal. Sustainable Cities and Society, 26: 438-446. https://doi.org/10.1016/j.scs.2016.04.015 
(57) Alchapar, N. (2014). Materiales de la envolvente urbana. Valoración de su aptitud para mitigar la isla de calor en ciudades de zonas áridas (Tesis doctoral). Universidad Nacional de Salta.

(58) Krüger, E., Pearlmutter, D., \& Rasia, F. (2010). Evaluating the impact of canyon geometry and orientation on cooling loads in a high-mass building in a hot dry environment. Applied Energy, 87(6): 2068-2078. https://doi.org/10.1016/j. apenergy.2009.11.034

(59) Ali-Toudert, F. \& Mayer, H. (2006). Numerical study on the effects of aspect ratio and orientation of an urban street canyon on outdoor thermal comfort in hot and dry climate. Building and Environment, 41(2): 94-108. https://doi. org/10.1016/j.buildenv.2005.01.013

(6o) Alchapar, N., Pezzuto, C., Correa, E. \& Labaki, L. (2017). The impact of different cooling strategies on urban air temperatures: the cases of Campinas, Brazil and Mendoza, Argentina. Theoretical and Applied Climatology, 130: 35-50. https:// doi.org/10.1007/s00704-016-1851-5

(61) Terando, A. J., Youngsteadt, E., Meineke, E. K., \& Prado, S. G. (2017). Ad hoc instrumentation methods in ecological studies produce highly biased temperature measurements. Ecology and Evolution, 7(23): 9890-9904. https://doi. org/10.1002/ece3.3499

(62) Huwald H., Higgins C. W., Boldi M.O., Bou-Zeid E., Lehning M., \& Parlange M. B. (2009). Albedo effect on radiativeerrors in air temperature measurements. Water Resources Research, 45(8): Wo8431. https://doi.org/10.1029/2008WRoo7600

(63) Richardson S. J., Brock F. V., Semmer S. R., \& Jirak C. (1999). Minimizing errors associated with multiplate radiation shields. Journal of Atmospheric and Oceanic Technology, 16(11): 1862-1872. https://doi.org/10.1175/15200426(1999)016<1862:MEAWMR>2.0.CO;2 